\title{
FABRICATION AND CHARACTERIZATION OF IC-COMPATIBLE MULTILAYER INTERFERENCE FILTERS
}

\author{
G. de Graaf, A. Emadi and R. F. Wolffenbuttel \\ Faculty EEMCS, Department ME/EI, \\ Delft University of Technology, \\ Mekelweg 4, 2628 CD, \\ Delft, The Netherlands
}

\section{Introduction}

This paper reports the design and characterization of IC-compatible Multilayered Fabry-Pérot Midinfrared optical filters. The filters have been designed for application in Mid-infrared Spectrometry [1]. An important class of microspectrometers in IC technology is based on arrays of optical filters and detectors composed of IC-compatible materials with proper optical properties over the spectral range of interest [2]. An IC-processed wafer with circuits and a micro-fabricated array of IR detectors can be used as a generic platform and accommodated to suit a particular application by post-process sputtering of layers of appropriate thickness. The filters have been fabricated as layered thin-film stacks of $\mathrm{SiO}_{2}$ and polySilicon. A set of filters covering the range from $2.2 \mu \mathrm{m}$ to $3 \mu \mathrm{m}$ with a typical HPBW of $30 \mathrm{~nm}$ and another series tuned at $3.8 \mu \mathrm{m}$ to $4.3 \mu \mathrm{m}$ have been designed, fabricated and measured. Suitable samples have been tested for application as optical filters for NDIR infrared gas sensing applications[3].

\section{Filter Design}

A Fabry-Pérot optical filter consists of an optical resonance cavity in between two parallel mirrors [4], as shown in Figure 1a. The mirrors are composed of a stack of quarter-wavelength layers with a high $\left(n_{H}\right)$ and a low $\left(n_{L}\right)$ refractive index, as depicted in Figure $1 \mathrm{~b}$.

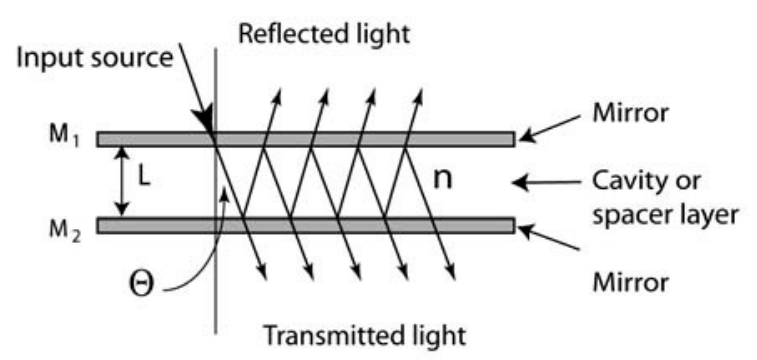

a.)

Figure 2. a.) Basic Fabry-Pérot device.

b.) Multilayer Fabry-Pérot filter.

\begin{tabular}{|ll|}
\hline $\mathrm{H}$ & $\mathrm{n}_{\mathrm{H}}$ \\
\hline $\mathrm{L}$ & $\mathrm{n}_{\mathrm{L}}$ \\
\hline $\mathrm{H}$ & $\mathrm{n}_{\mathrm{H}}$ \\
\hline $\mathrm{L}$ & $\mathrm{n}_{\mathrm{L}}$ \\
\hline $\mathrm{L}$ & $\mathrm{n}_{\mathrm{L}}$ \\
\hline $\mathrm{H}$ & $\mathrm{n}_{\mathrm{H}}$ \\
\hline $\mathrm{L}$ & $\mathrm{n}_{\mathrm{L}}$ \\
\hline $\mathrm{H}$ & $\mathrm{n}_{\mathrm{H}}$ \\
\hline
\end{tabular}

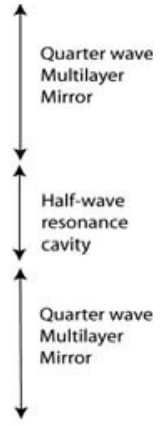

b.)

If many layers are used, the reflectivity can become very high. For large numbers of layer pairs $N$ the reflectivity $R$ can be approximated by [5]: $\quad R=1-4\left(\frac{n_{L}}{n_{H}}\right)^{N}$. The transmission of a Fabry-Pérot device is at a sharp maximum if constructive interference occurs in the spacer layer i.e. if the optical thickness of the spacer layer equals half the wavelength. Therefore by changing the thickness of only the center layer, the tuned wavelength is changed. Poly-Silicon $\left(n_{H}=3.4\right)$ and $\mathrm{SiO}_{2}\left(n_{L}=1.3\right)$ thin film layers have been used as the high- $n$ the low- $n$ material. Low temperature deposition of these materials allows integration in many integrated circuit or MEMS processes. (Poly)Silicon has a low absorption at wavelengths beyond $\lambda=1.2 \mu \mathrm{m}$ and can be considered transparent above $\lambda=1.5 \mu \mathrm{m}$ [6]. $\mathrm{SiO}_{2}$, however, is transparent in the visible range and absorption increases sharply beyond $\lambda=3 \mu \mathrm{m}$ [7]. Since the total thickness of the $\mathrm{SiO}_{2}$ layers is small can be used for filters up to around $\lambda=8 \mu \mathrm{m}$ if some loss in the resonance peak can be accepted. 
Thin-film optics software packages TFCalc 3.3 [8] and MacLeod [9] have been used for simulation and optimization of layers in optical filters. The filters can be optimized for either maximum Free Spectral Range (FSP) or resolution for a given number of layers. Figure 2 shows simulated response of an array of FP filters designed with 10 layers (including the substrate) in which the thickness of the middle $\mathrm{SiO}_{2}$ layer ranges from 660 to $1020 \mathrm{~nm}$ with $20 \mathrm{~nm}$ increments, resulting in 20 resonance peaks from $2.2 \mu \mathrm{m}$ to $2.86 \mu \mathrm{m}$ with $30 \mathrm{~nm}$ wavelength increments. Table 1 shows the calculated layer thicknesses for the $2.5 \mu \mathrm{m}$ series.

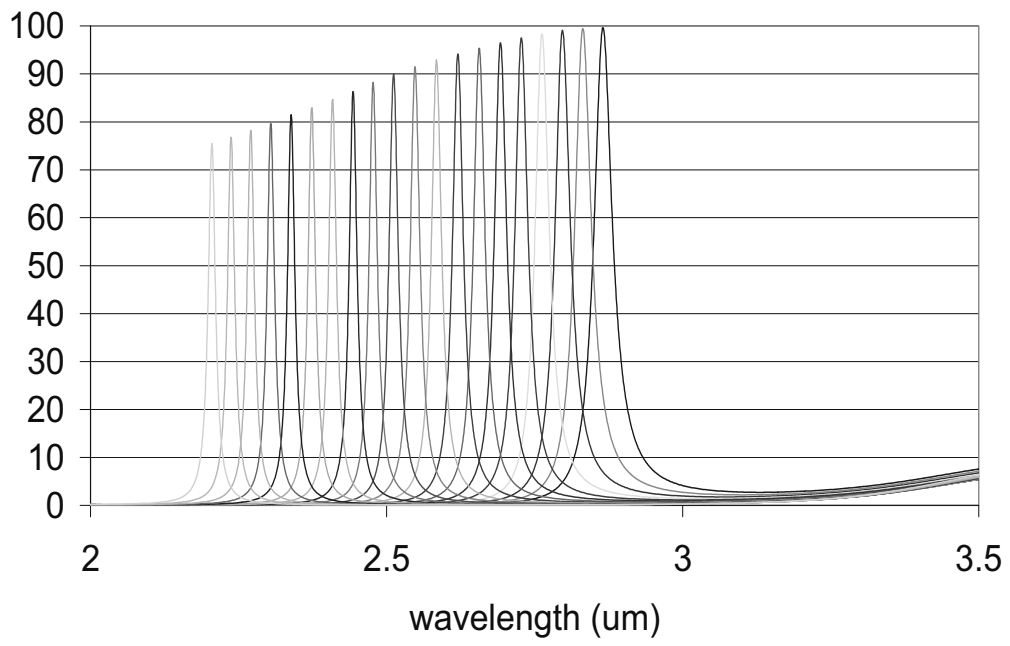

Figure 2. Simulated transmission of the designed filters.

Table 1. Designed values of the FP filter layer thicknesses.

\begin{tabular}{|c|c|c|}
\hline Layer number & Material & $\begin{array}{c}\text { Thickness } \\
(\mathrm{nm})\end{array}$ \\
\hline 1 & $\mathrm{Si}($ Substrate) & - \\
\hline 2 & $\mathrm{SiO}_{2}$ & 321 \\
\hline 3 & $\mathrm{PolySi}$ & 144 \\
\hline 4 & $\mathrm{SiO}_{2}$ & 463 \\
\hline 5 & $\mathrm{PolySi}$ & 216 \\
\hline 6 & $\mathrm{SiO}_{2}$ & $660-1020$ \\
\hline 7 & $\mathrm{PolySi}$ & 216 \\
\hline 8 & $\mathrm{SiO}_{2}$ & 463 \\
\hline 9 & $\mathrm{PloySi}$ & 144 \\
\hline 10 & $\mathrm{SiO}$ & 321 \\
\hline 11 & $\mathrm{PolySi}$ & 116 \\
\hline
\end{tabular}

\section{Fabrication}

A FHR MS 150 Sputter system has been used to deposit the layers continuously on $10 \times 10 \mathrm{~mm}^{2}$ samples from a 6 inch double-polished silicon wafer. The system can handle substrates up to $150 \mathrm{~mm}$ and is equipped with four magnetron sputter cathodes and an optical spectrometer for on-line thickness measurement. The deposition of the two materials has been characterized and calibrated by measuring layer thickness and uniformity over the wafer. In the beginning, two test deposition runs of $\mathrm{SiO}_{2}$ and PolySilicon on two separate 6 inch wafers are done. The reason for this is to characterize the materials, calibrating the deposition rate and verifying thickness variations over the whole deposition area. Figure 3a and $3 \mathrm{~b}$ show the thickness variations of deposited Silicon and $\mathrm{SiO}_{2}$ over 149 points on a 6 inch wafer. The thicknesses were measured by ellipsometry. 

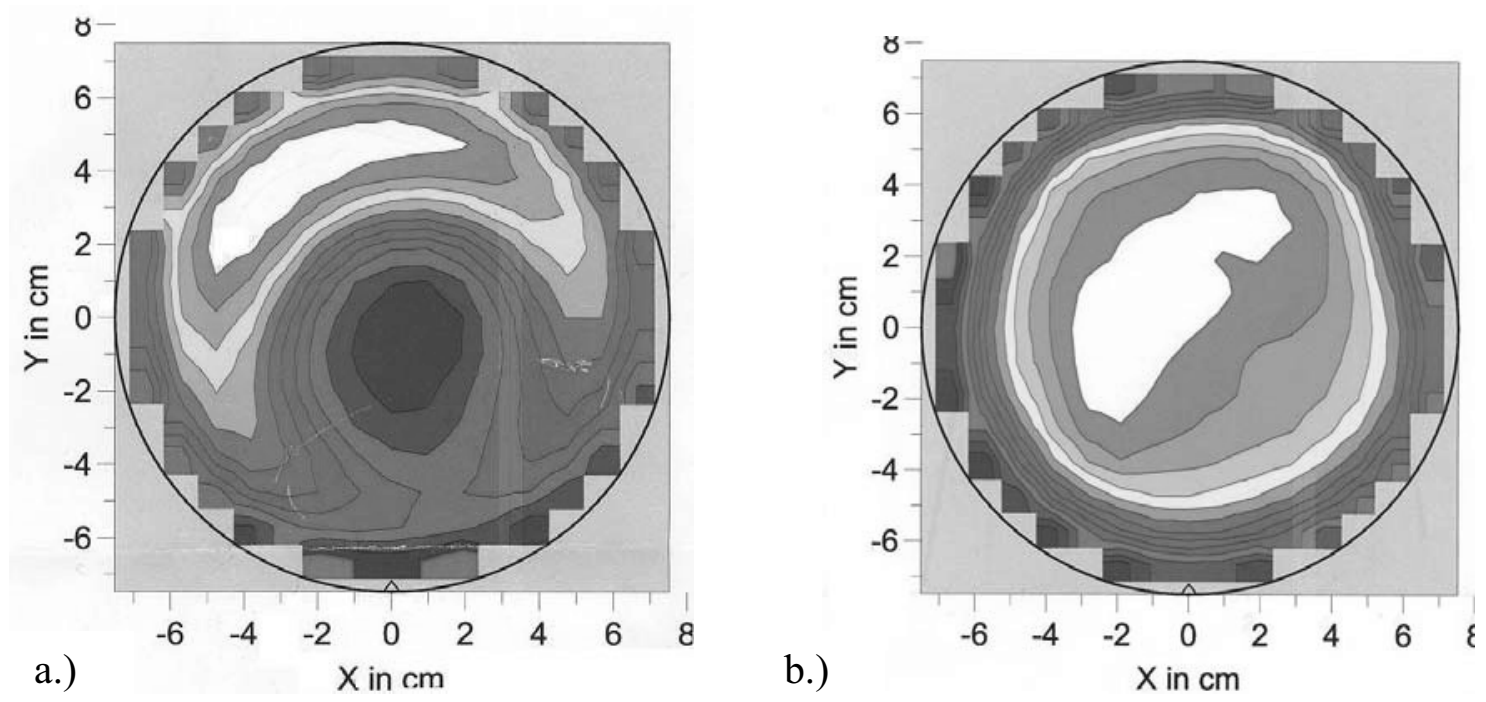

Fig.3 a.) Thickness variation of Sputtered Silicon over a 6 inch wafer.

b.) Thickness variation of Sputtered $\mathrm{SiO}_{2}$ over a 6 inch wafer.

For Sputtered Silicon the thickness varies from $187 \mathrm{~nm}$ to $218 \mathrm{~nm}$ with initial target thickness of $200 \mathrm{~nm}$. This means that there can be almost $\pm 10 \%$ error in thickness of sputtered Silicon over the wafer. For sputtered $\mathrm{SiO}_{2}$ the variation of layer thickness is between $401 \mathrm{~nm}$ and $481 \mathrm{~nm}$ with initial aimed thickness of $400 \mathrm{~nm}$. Ellipsometry measurements were done at three stages on the samples; after deposition of the first stack of layers, after depositing the middle SiO2 layer and after deposition of the final layers. Ellipsometric data analysis software has been used to find actual thicknesses of the layers.

\section{Measurements}

Filters have been measured with a Fourier Transform Spectrometer which can provide a spectral resolution of $2 \mathrm{~nm}$. Figure 3 shows the measured response of the some of the filtersof the $2.5 \mu \mathrm{m}$ series.

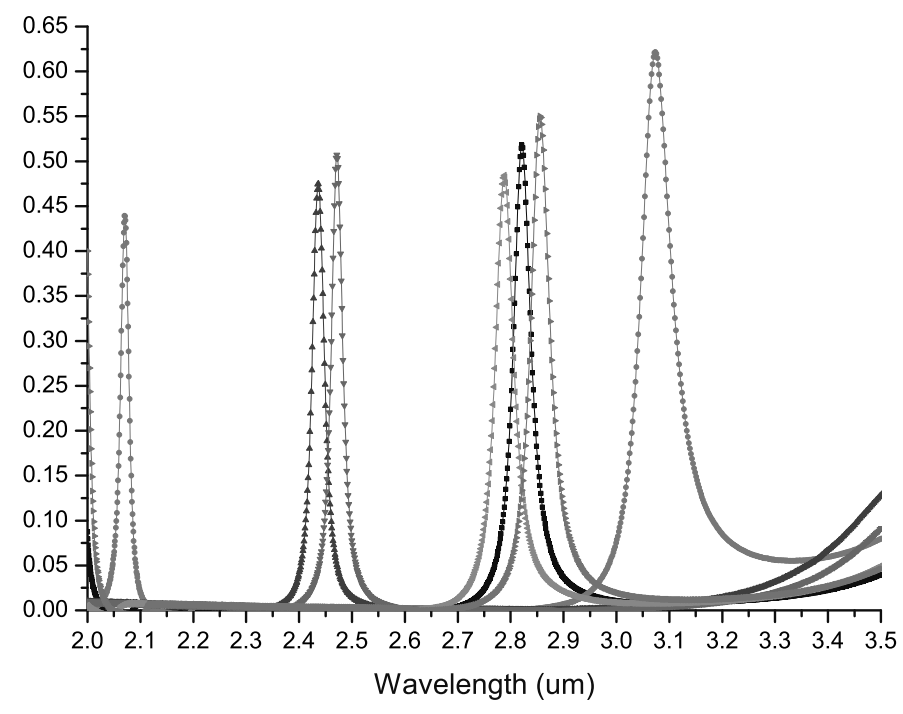

Figure 4. Measured infrared transmission of the $2.5 \mu \mathrm{m}$ Fabry-Pérot filters.

Some shift in the peak response of the filters has been observed, due the inaccuracy of the thickness in the spacer layer. The HPBW of the filters is measured to be $35 \mathrm{~nm}$. Based on the results and experience of the first batch an improved second series with a centre wavelength around $4 \mu \mathrm{m}$ has been fabricated. The results are shown in Figure 5. 


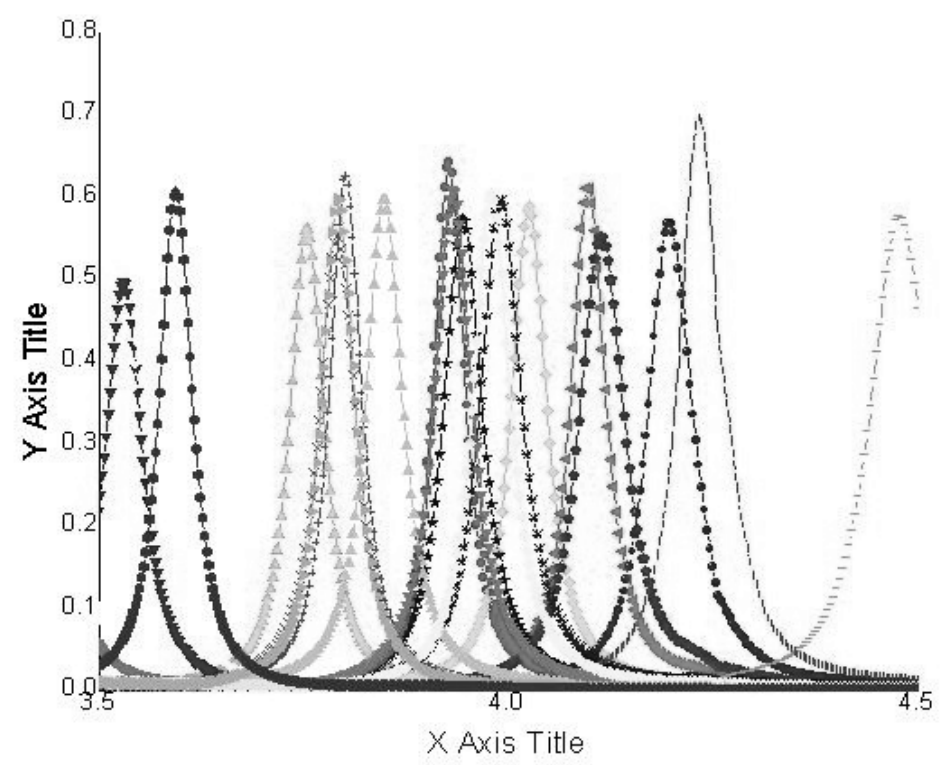

Figure 5. Measured transmission of the $4 \mu \mathrm{m}$ Fabry-Pérot filters.

NDIR spectrometers have a simple and straightforward optical path, enabling low-cost and are typically used as dedicated systems in automotive applications [10] or in portable instruments [11]. The $4 \mu \mathrm{m}$ filters have been tested in the NDIR spectrometer setup shown in Figure 6. A pulsed thin film thermal infrared emitter has been used as the mid-infrared source. The infrared beam is collimated in the sample chamber and focused into the filter/detector by a set of $90^{\circ}$ spherical mirrors. The infrared thermopile detector signal is read by a Stanford Research SR830 Lock-in amplifier operating at a chopper frequency of $15 \mathrm{~Hz}$. Different gases could be detected by measuring the response of different filters. Quantative measurements have not been performed because no calibrated instruments for gas concentration measurements were available.

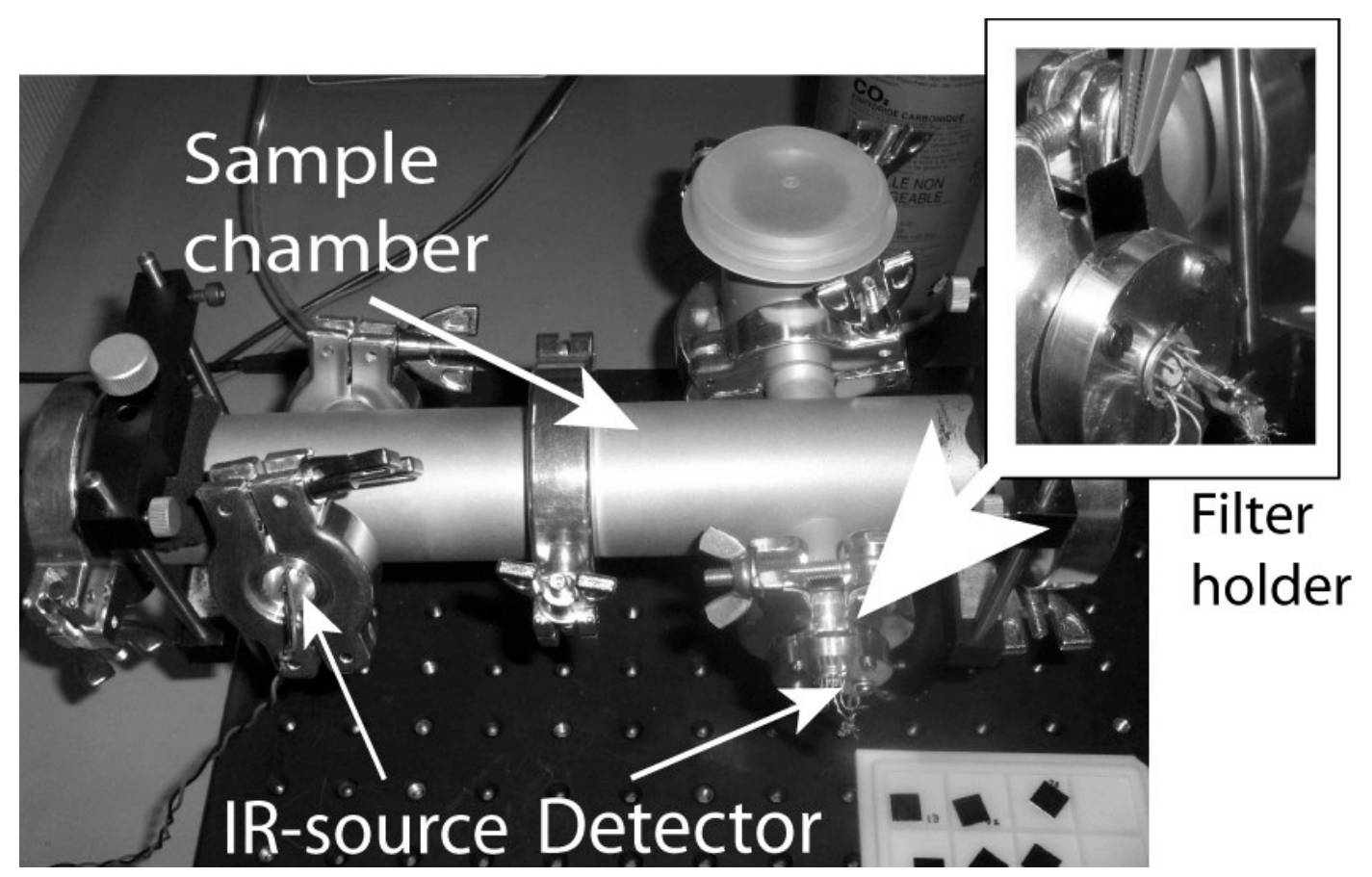

Figure 6. Measurement setup for NDIR gas detection using the fabricated filters. 


\section{CONCLUSIONS}

Narrowband silicon-compatible bandpass Fabry-Pérot filters have been fabricated using multilayer thin film mirrors. Despite some shift in the response of the filters, sets of narrow band filters have been fabricated in the 2.4-3.2 $\mu \mathrm{m}$ and $3.0-4.5 \mu \mathrm{m}$ infrared spectral range on $10 \times 10 \mathrm{~mm}^{2}$ silicon substrates. The FWHM of the filters is measured to be $35 \mathrm{~nm}$. More accurate calibration of the deposition process from the results and thicknesses achieved by ellipsometric analysis can help to further improve control of the thickness of the layers. From the simulations shown, it can be concluded that filters of 8 layers or more can provide adequate filtering for many mid-infrared applications. The most critical part in the fabrication of thin film filters is precise thickness control. Theoretically it can be possible to control the thicknesses very accurate if each filter die is fabricated separately, however this is not practical or economical.

The filters are intended for use in Non-dispersive infrared ( NDIR ) mid-infrared spectrometers, where the transmission peaks of the optical filters should be designed to coincide with the absorption maxima and minima of a sample. A relatively simple complete spectrometer system can be fabricated by the integration of the discussed IC compatible Fabry-Pérot filters, with an integrated infrared thermal thin film source and an integrated thermopile detector array. A setup for gas measurement has been built for testing this principle.

\section{REFERENCES}

[1] H. Gunzler, H-U. Gremlich, "IR Spectroscopy: An Introduction", ISBN 3-527-28896-1, Wiley-VCH Verlag GmbH, 69469 Weinheim, Germany, 2002.

[2] R.F. Wolffenbuttel, "State-of-the-art in integrated optical microspectrometers", IEEE Transactions on Instrumentation and Measurement, , 2004, Vol. 53 No. 1, Page(s):197-202.

[3] D. W Hill, "Non-dispersive infra-red gas analysis in science, medicine and industry",222 pages, Publisher: Hilger (1968) ISBN-13: 978-0852740903.

[4] M. Vaughan, "The Fabry-Perot interferometer: history, theory, practice and applications", Adam Hilger, 1989.

[5] A. Thelen, "Design of Optical Interference Coatings", New York: McGraw-Hill, 1989.

[6] A. Tabata, N. Matsuno, T. Mizutani T., Y. Suzuoki, "Optical properties and structure of SiO2 films prepared by ion-beam sputtering", Thin Solid Films, Elsevier, Volume 289, Number 1, 30 November 1996 , Page(s): 84-89(6).

[7] Y. Laghla and E. Scheid, "Optical study of undoped, B or P-doped polysilicon", Thin Solid Films, Volume 306, Issue 1, August 1997, Page(s): 67-73.

[8] http://www.sspectra.com, Software Spectra Inc.,"TFCALC 3.3l”, 14025 N.W. Harvest Lane, Portland, OR 97229 , USA.

[9] http://www.thinfilmcenter.com, "The Essential MacLeod", Thin Film Center Inc., 2745 E Via Rotonda, Tucson, AZ 85716-5227, USA.

[10] R. Frodl, T. Tille, "A High-Precision NDIR CO2 Gas Sensor for Automotive Applications", Sensors Journal, IEEE, Volume 6, Issue 6, Dec. 2006 Page(s):1697 - 1705.

[11] D. Sebacher, "Non-dispersive infrared absorption monitors for trace gases", Inpared Method for Gaseous Measurements, Marcel Dekker, New York, 1985, Ch. 6, Page(s) 247-274. 